\title{
An Experimental Study of Voice-Coil Driven Synthetic Jet Propulsion for Underwater Vehicles
}

\author{
AM Polsenberg-Thomas ${ }^{1}$, Joel Burdick ${ }^{1}$, Kamran Mohseni ${ }^{2}$ \\ ${ }^{1}$ Engineering and Applied Science, Caltech, Pasadena, CA 91125 \\ ${ }^{2}$ Dept. of Aerospace Engineering Sciences, University of Colorado, Boulder, CO 80309 \\ ampolsen@caltech.edu
}

\begin{abstract}
This paper investigates the thrust and flow structures produced by submerged synthetic jet actuators. Inspired by the propulsion methods of many sea creatures, such as jellyfish, squids, and salps; synthetic jets use vortex rings to create a net thrust. To assess the potential usability of these thrusters for propulsion and maneuvering of small underwater vehicles, a range of synthetic jet thruster prototypes were built, and both flow visualization and thrust measurement experiments were executed. Based on the experimental results obtained from these models, we discuss the feasibility of using these thrusters on small, slow, but maneuverable vehicles.

Index Terms - synthetic jet, AUV, vortex ring, optimization, propulsion
\end{abstract}

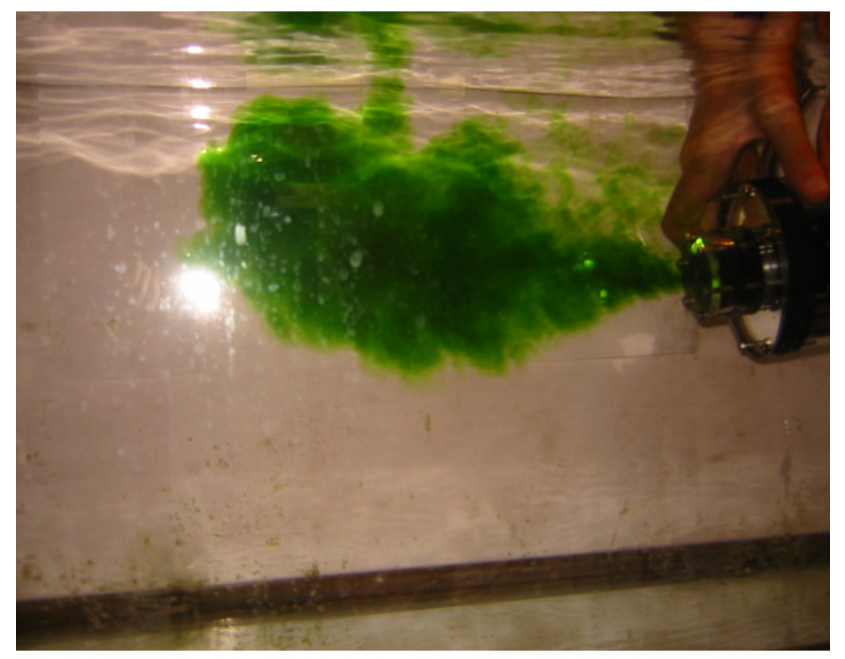

Fig. 1. Dye visualization of the wake produced by a voice coil driven synthetic jet

\section{INTRODUCTION}

Currently, AUVs (Autonomous Underwater Vehicles) are most commonly used for large scale surveying and mapping projects. Virtually all AUVs are propelled by a single stern propeller. Additionally, propeller design and efficiency has been studied and improved upon for hundreds of years[1], [2]. This configuration, while excellent for cruising situations, does not allow these vehicle to easily hover, and thus they are not well suited for slow speed tasks involving tight maneuvering and station keeping. As more and more new uses are being found for unmanned underwater vehicles, it becomes interesting to investigate how alternative propulsion schemes can add to the capability of
AUVs. Slow speed maneuverability can be crucial for tasks such as tracking slow moving animals such as jellyfish and larvaceans, and investigating enclosed or treacherous areas where excellent maneuverability is required. The goal of this work is to look at synthetic jets as a potential slow speed propulsor well suited to handle tasks like biological tracking and exploration of hazardous environments.

One major weakness of using propellers (ducted or unducted) is that they typically protrude from the body of the vehicle. The thrusters, and any other protruding parts, are at risk of breaking off. In particular, during launching, recovery and manuevering in enclosed areas such as caves or shipwrecks, these protrusions are a huge liability. Additionally, when the vehicles are used to inspect or track animals, creatures have been killed by propellers.

In addition to desiring a propulsion system which will allow for highly maneuverable, controllable low-speed travel, we believe that the following are equally important criteria:

- Minimal moving parts to increase mechanical robustness

- No parts protruding from the hull of the vehicle to become entangled with surrounding environment, or the biological creatures being tracked by the vehicle

- Minimal volume, to allow more payload, which is especially crucial in small vehicles

- Off the shelf components, thereby enabling low cost small vehicles

\section{A. Biological Inspiration}

Pulsatile jet propulsion, as seen in squids, salps, and jellyfish, may offer a propulsion scheme that meets many of these design goals [3], [4], [5], [6], [6], [7], [8]. These, and other animals, propel themselves by pulsing water out of an orifice in their body. As the fluid exits the orifice, it rolls into a vortex ring. The pulsatile jet created by these rings has been shown to produce a greater average thrust than that of a steady jet with the same mass flux [8]. As Lane points out, the advantages of this approach were discovered by nature: "Ages before man discovered jet-propulsion, cephalopods were jetting through primeval seas [9]."

Nature suggests that this style of propulsion may offer advantages for smaller vehicles operating at modest speeds. Jellyfish and salps are relatively small, and while there are some very large species of squid, the majority of squid species are small [10]. (O'Dor proposes that the possible 
geometric and energetic constraints may play a role in explaining the prevalence of smaller squid [10]) Whether or not these constraints will apply to a synthetic jet propelled vehicle remains to be seen, but this size distribution shown in squids should not be ignored and has led us to begin our investigation into small vehicles.

\section{B. Pulsatile Jet Propulsion via Synthetic Jets}

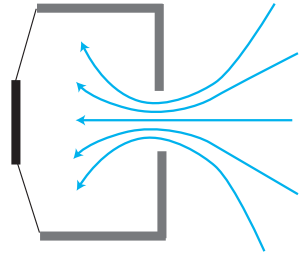

A

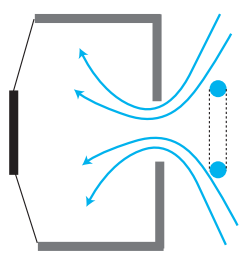

C

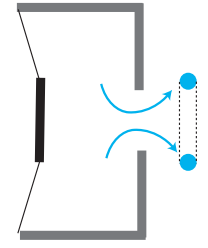

B

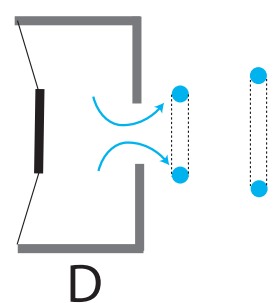

Fig. 2. The stages of synthetic jet operation: (A) initial inflow, (B) initial outflow, (C) subsequent inflow, (D) subsequent outflow

Zero net mass flux jets, also known as synthetic jets, are one means to generate pulsatile jet fluid flows. Synthetic jets have long been used in the aerospace industry for flow control [11], [12], [13], [14]. A synthetic jet consists of a chamber with a vibrating membrane at one end and an orifice on the other. During the membrane's in-stroke, fluid is sucked in through the orifice. During the out-stroke, fluid is ejected through the orifice, creating a vortex ring. The process repeats. A series of rotating rings entrain fluid, creating a jet. This is illustrated in Figures 2 and 3 . Krueger has shown the potential thrust augmentation benefits of pulsatile jets [15], [16], [17].

The use of synthetic jets for underwater vehicle propulsion was first proposed by Mohseni [18], [19], [20]. In these works, Mohseni and collaborators investigated the use of solenoids and cams to actuate the synthetic jet stroke. In

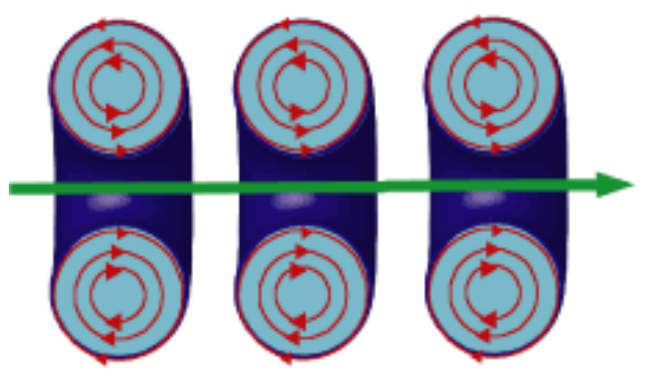

Fig. 3. Vortex Rings Forming a Jet

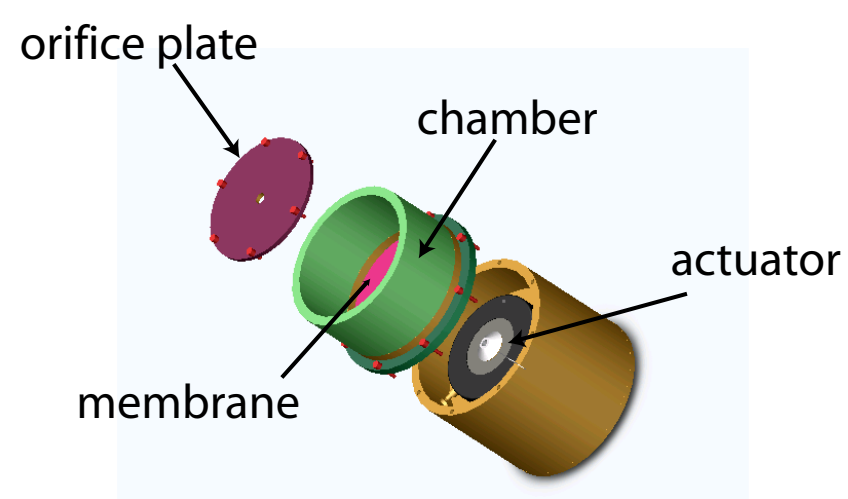

Fig. 4. Schematic diagram of a synthetic jet prototype

this paper, we investigate a synthetic jet design based on a voice coil actuator. This paper updates our prior work on this type of actuator design [21]. We note that this earlier paper [21] did not properly recognize prior work by Kamran Mohseni on the use of synthetic jets for underwater vehicle propulsion.

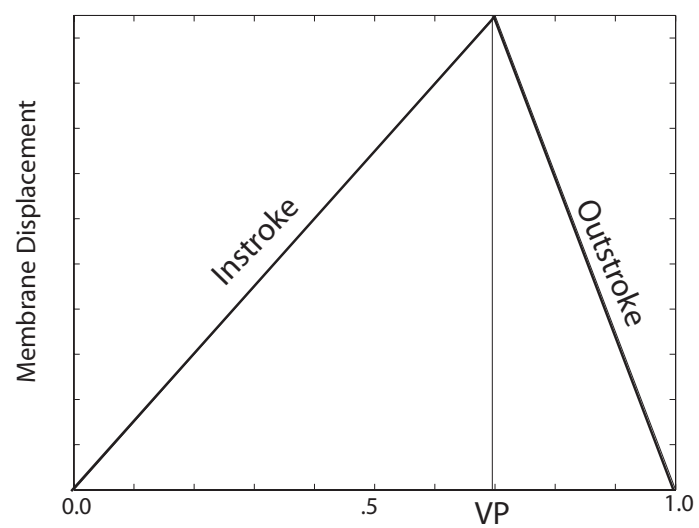

Fig. 5. Membrane velocity profile (VP). VP represents the instroke fraction of a single membrane cycle. (For example, a VP of .6 means that the instroke accounts for $60 \%$ of the cycle time.

A synthetic jet can be built by taking a hollow cylindrical chamber and placing a vibrating membrane at one end and a lid with an orifice in it at the other end. We designed modular prototypes so that elements such as orifice shape and diameter, chamber length, and actuation device can easily be changed to investigate the effects of these design parameters on performance.

There are many possible ways to oscillate the membrane. We have built two different voice coil actuated prototypes. One prototype is actuated by using a Bruel and Kjael 4810 minishaker (designed to vibrate small objects). The second prototype is built using a voice coil from a consumergrade audio speaker. For these prototypes there are two variable parameters: the frequency of membrane forcing, and the velocity profile of the membrane's displacement. In the experimental data presented below, the membrane's velocity profile was defined to be a periodic sawtooth 
function with a fixed amplitude. The profile parameter $V P$, ranging from 0 to 1 , is the point at which the membrane switches direction (see Figure 5). (Values of $V P$ smaller than 0.5 refer to profiles where the membrane pushes out slower than it is pulled in.)

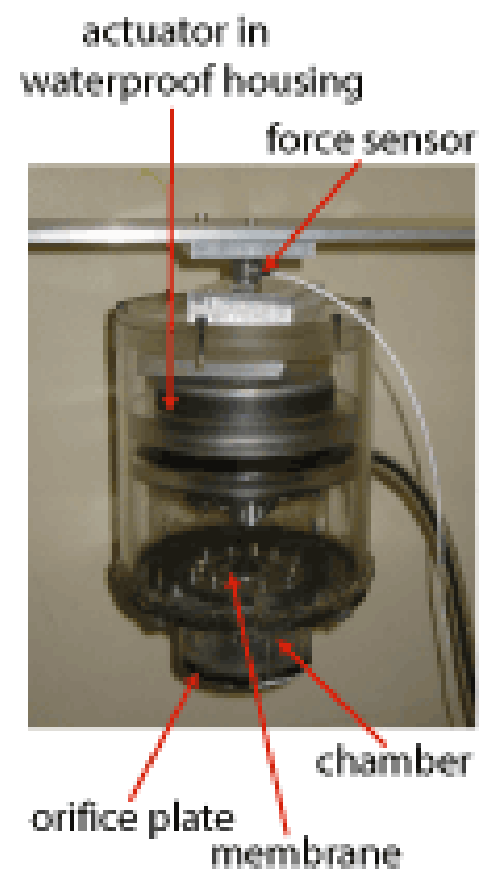

Fig. 6. Force measurement setup (pictured with speaker coil actuated synthetic jet)

For the speaker coil driven synthetic jet, which will be discussed in detail in Section II, the following are the values for the variables shown in Figure 7.

$$
\begin{gathered}
\text { delta }=3 \mathrm{~mm} \\
d_{\text {disk }}=25.4 \mathrm{~mm} \\
d_{\text {chamber }}=50.8 \mathrm{~mm} \\
d_{\text {orifice }}=15.875 \mathrm{~mm}
\end{gathered}
$$

\section{ForCE MEASUREMENT}

To measure the average net thrust produced by the synthetic jet prototypes, the jets were attached to a strain gauge force sensor (Interface Advanced Force Measurement Part WMC-5) as is shown in Figure 6. The prototype to be tested screws onto one end of the sensor while the other end is rigidly attached to a stand. The setup is submerged and forces are then measured through a Measurement Computing Corporation PC-CARD-DAS16 analog data acquisition card.

Using the Bruel and Kjael minishaker prototype, average thrusts of up to $0.25 \mathrm{~N}$ were measured. However, due to the high price of the minishaker, we also developed a protoype

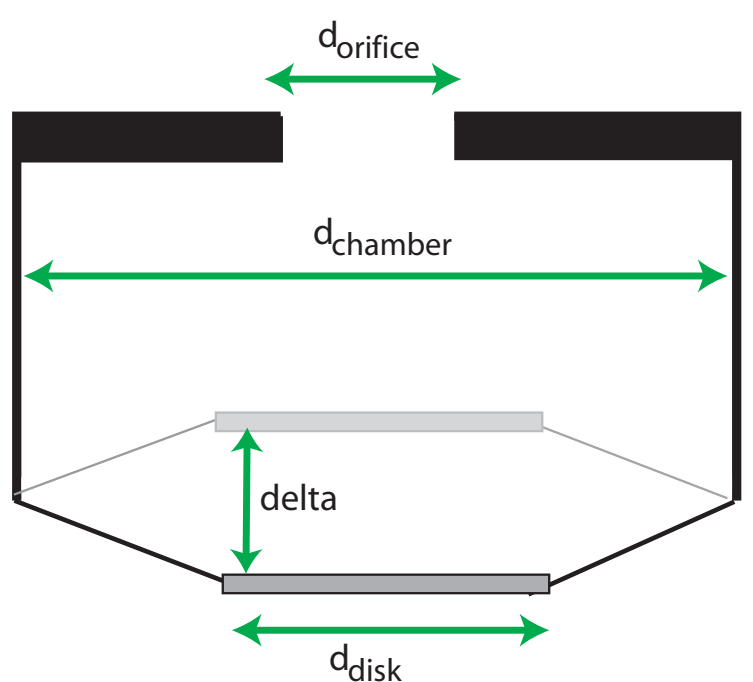

Fig. 7. Speaker coil driven synthetic jet

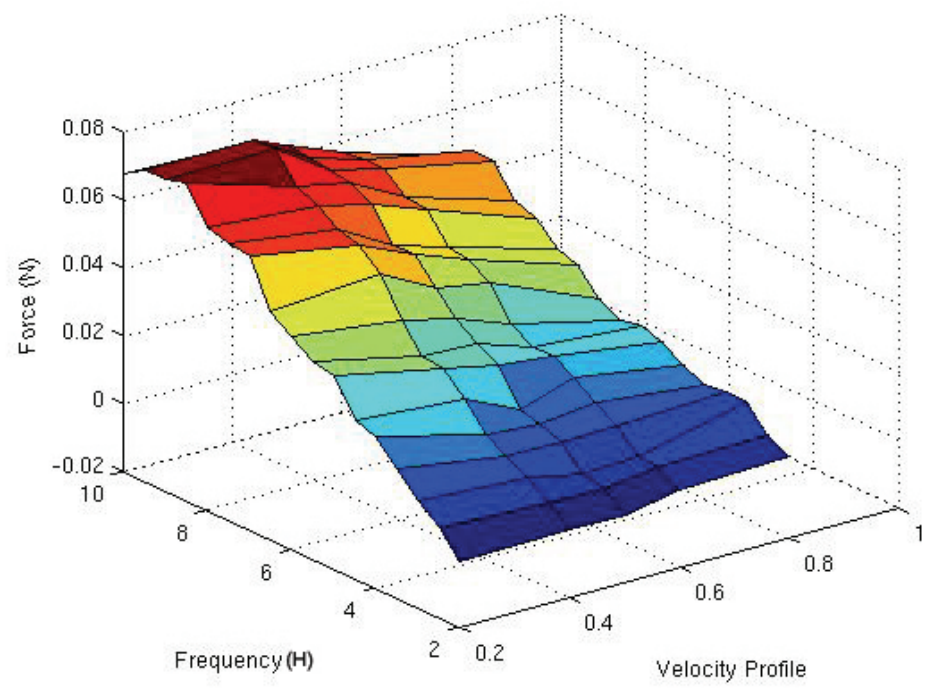

Fig. 8. Recorded force (for prototype actuated by off-the-shelf speaker coil) as a function of forcing frequency and outstroke velocity profile

that employs an off-the-shelf voice coil actuator (as one of the goals of this project is to design a low cost thruster).

As can be seen in the results for the consumer-grade voice coil (Figure 8), frequency and velocity profile have a significant effect on the produced thrust. In an actuation region of 1-10 Hertz, increased frequency leads to an increase in force. Additionally, in this region, a smaller velocity profile (which corresponds to membrane forcing profiles with a slow outstroke and a fast instroke) produced a greater thrust.

In Figure 9, the same data is shown normalized by outstroke velocity. This normalized data is particularly useful, as it accounts for the fact that higher frequency cases have higher average velocity, which skews the force 


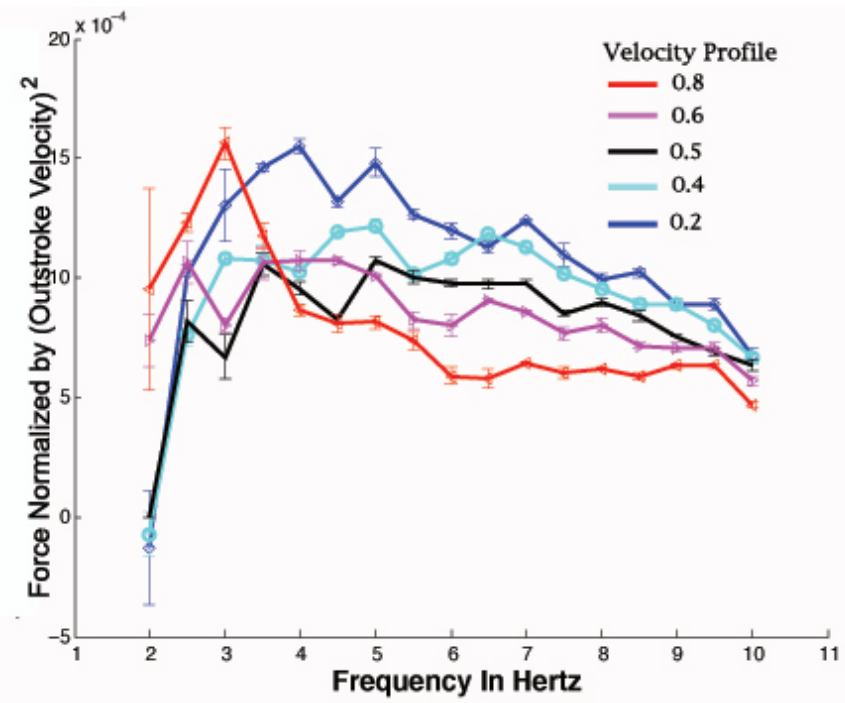

Fig. 9. Recorded average force normalized by outstroke velocity

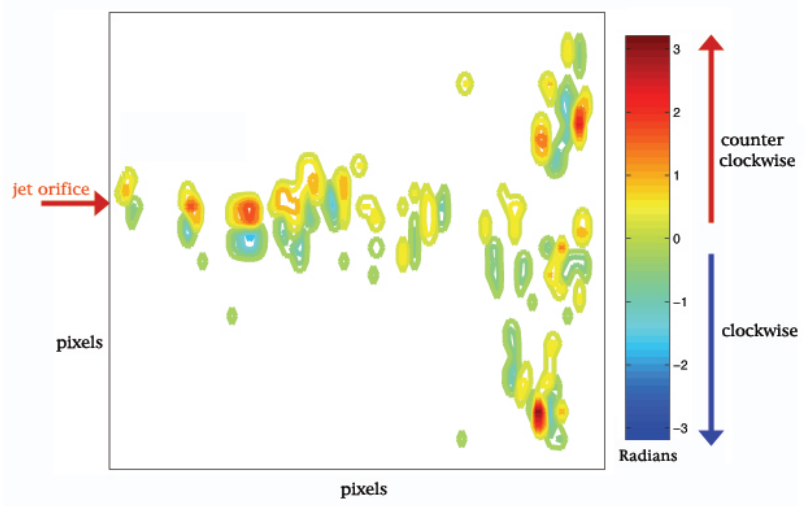

Fig. 10. Cross-Sectional image of vortex ring jet (pulsed at $10 \mathrm{Hertz}$ ) obtained by DPIV. The jet flows from left to right.

measurement in their favor. While high frequencies may produce a higher thrust, it is at a higher energy cost. After normalization, our preliminary data suggets that lower frequencies (approximately 2-5 Hertz) may provide the most efficient propulsion.

\section{FLOW VISUALIZATION}

As most of the literature on synthetic jets is concerned with jets in air, there is much to be learned about the flow structures created by the jets in water. Early stages of this project have been focused on gathering flow visualization data, both qualitative and quantitative. The qualitative data was gathered by using a high speed camera to capture the wake created by the jet using food coloring. It is evident from these early efforts that well defined vortex rings and a coherent jet structure is formed. (See Figure 1.)

For quantitative data on the fluid flow induced by a synthetic jet, Digital Particle Image Velocimetry (DPIV) [22] was performed for trials using different jet parameters. A pulsed $150 \mathrm{~mW}$ New Wave Gemini Nd:YAG laser was used, with a time step of $10 \mathrm{~ms}$ between frames. The water was seeded with 14 micron reflective particles. The data was processed by using a window size of $32 \times 32$ pixels with an overlap of 16 pixels. This processing allows for the estimation of overall velocity and vorticity fields. For this paper, DPIV was performed by using laser sheets perpendicular to the jet. This allows us to see crossSections of the vortex rings, as seen in Figure 10. Vortex rings, seen as vortex pairs in this cross-Sectional image, are clearly visible. Notice that the jet assumes a more complex structure, resulting in blooming of the jet, in the far field.

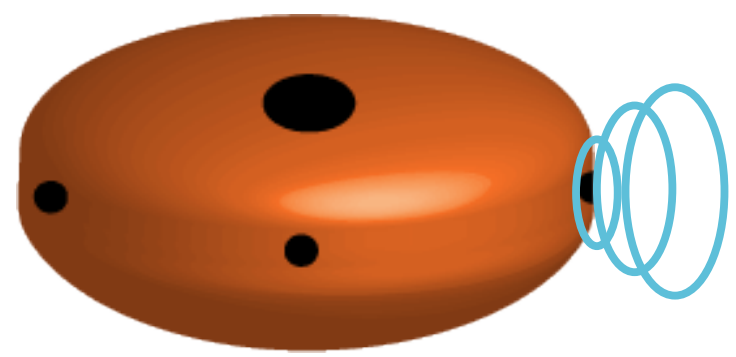

Fig. 11. Illustration of proposed vehicle shape.

\section{Vehicle Design Considerations}

This work seeks to demonstrate that low cost synthetic jets can provide adequate propulsion and maneuvering forces for small underwater vehicles moving at modest speeds. Since one motivation for this project is to design a vehicle capable of tracking a very slow moving sea creature (such as a larvacean), our initial goal is to create a vehicle which can reach speeds of approximately $5 \frac{\mathrm{cm}}{\mathrm{s}}$. (A potentially interesting hull shape would be an oblate spehoid, as shown in Figure 11. This will allow for more vertical stability, which would be beneficial for tasks which require the vehicle to hover. The drag for this "flying saucer," shape will be less than the drag of a sphere. Thus the following calculations are a worst case scenario.) Given the dimensions of our current thruster prototypes, a reasonable diameter for the vehicle (in the horizontal plane) is 0.25 meters.

Our initial concern was whether a single synthetic jet would be sufficient to overcome the drag of such a vehicle. The following calculations are done for a "worst case scenario" spherical vehicle, assuming steady flow:

$$
\text { Drag }=C_{D} \frac{1}{2} U^{2} \rho S
$$

where $U$ is the vehicle speed, $\rho$ the density of water, and $C_{D}$ is the drag coefficient. The drag coefficient can be found as a function of the ambient flow's Reynolds number, $R e=U D / \nu$, where $D$ is the vehicle diameter and $\nu$ is the kinematic viscosity of water.

As can be seen in Figure 12, the minishaker actuated synthetic jet is sufficient for propelling the spherical vehicle at speeds of well over $10 \frac{\mathrm{cm}}{\mathrm{s}}$, while the consumer grade speaker coil can propel the vehicle at speeds up to $8 \frac{\mathrm{cm}}{\mathrm{s}}$ . Note that for the thruster configurations shown in Figure 
11, additional forces can be generated by simultaneous actuation of two thrusters, thus increasing the speed of the vehicle.

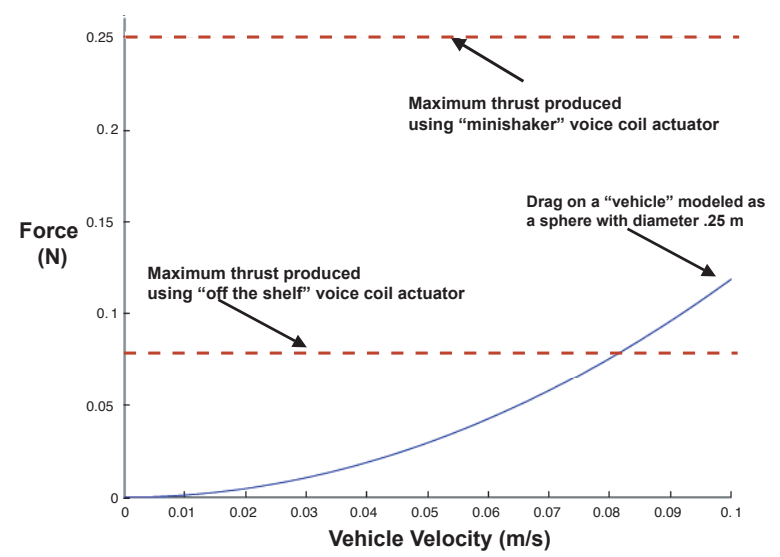

Fig. 12. Comparison of forces produced by prototype synthetic jet thrusters and the drag of a spherical "vehicle."

\section{CONCLUSION}

There are many potential advantages to using synthetic jets as propulsors for AUVs. The compact size of the thrusters, single moving part, single seal and absence of protruding elements make them ideal for operation in complex environments and in very close proximity to small, slow moving creatures. While small, the forces they produce are sufficient for the vehicle scale that we are considering. Finally, the wake structures generated by synthetic jets are likely to be less obtrusive for underwater surveillance of biological organisms.

\section{ACKNOWLEDGMENT}

This work could not have been accomplished without the assistance of Professor John Dabiri, Dr. Michele Milano and Professor Mory Gharib of Caltech. Caltech student Kathleen Fischer is responsible for much of the early DPIV done on these prototypes. We would also like to thank Caltech students Joanna Cohen, Aimee Eddins, Jesse Escobeda, Vicente Fernandez, Maxwell Grazier G'Sell, and Amish Patel for their assistance in the productions of the thruster prototypes. The first author was partially supported by a Department of Defense fellowship and a PEO Scholar Award. Additonal support was also provided by the National Science Foundation, grants NSF9402726 and NSF0413078.

\section{REFERENCES}

[1] R. Bachmayer, L. Whitcomb, and M. Grosenbaugh, "An accurate four-quadrant nonlinear dynamic model for marine thrusters: theory, and experimental validation," IEEE Journal of Oceanic Engineering, vol. 25, pp. 146-159, 2000.

[2] J. Kerwin, MIT Lecture Notes: Hydrofoils and Propellers 13.014, 2001.
[3] J. Dabiri, S. Colin, J. Costello, and M. Gharib, "Flow patterns generated by oblate medusan jellyfish: field measurements and laboratory analyses," Journal of Experimental Biology, vol. 208, no. 7, pp. 1257-1265, 2005.

[4] M. Nixon and J. Messenger, The Biology of Cephalopods. London: Academic press, 1977.

[5] R. O'Dor and D. Webber, "The constraints on cephalopods: why squid aren't fish," Canadian Journal of Zoology, vol. 64, pp. 1591$1605,1986$.

[6] J. Seikmann, "On a pulsating jet from the end of a tube, with applications to the propulsion of certain aquatic animals," Journal of Fluid Mechanics, vol. 15, pp. 399-418, 1963.

[7] E. Trueman, "Motor performance of some cephalopods," Journal of Experimental Biology, vol. 49, pp. 495-505, 1968.

[8] D. Weihs, "Periodic jet propulsion of aquatic creatures," Fortschritte der Zoologie, vol. 24, pp. 171-175, 1977.

[9] F. Lane, Kingdon of the Octopus: The Life History of the Cephalopoda. New York: Sheridan House, 1960.

[10] R. O'Dor and J. Hoar, "Does geometry limit squid growth?" ICES Journal of Marine Science, vol. 57, pp. 2-14, 2000.

[11] B. Smith and A. Glezer, "Vectoring and small-scale motions effected in free shear flows using synthetic jet actuators," AIAA Paper 97$0213,1997$.

[12] A. Glezer and M. Amitay, "Synthetic jets," Annual Review of Fluid Mechanics, vol. 34, pp. 503-529, 2002.

[13] R. James, J. Jacobs, and A. Glezer, "A round turbulent jet produced by an oscillating diaphragm," Physics of Fluids, vol. 8, no. 9, pp. 2484-2495, 1996.

[14] A. Crook, A. Sadiri, and N. Wood, "The development and implementation of synthetic jets for the control of seperated flow," in 17th Applied Aerodynamics Conference, Norfolk, VA, 1999.

[15] P. Krueger, "The significance of vortex ring formation and nozzle exit over-pressure to pulsatile jet propulsion," $\mathrm{Ph} . \mathrm{D}$. dissertation, California Institute of Technology, 2001.

[16] P. Krueger and M. Gharib, "The significance of vortex ring formation to the impulse and thrust of a starting jet," Physics of Fluids, vol. 15, no. 5, pp. 1271-1281, 2003.

[17] P. Krueger and A. Gharib, "Thrust augmentation and vortex ring evolution in a fully pulsed jet," AIAA Journal, vol. 43, no. 4, pp. 792-801, 2005.

[18] K. Mohseni, "Impulse extremization in vortex formation for application in low speed maneuvering of underwater vehicles," in 23rd International Conference on Offshore Mechanics and Arctic Engineering, Vancouver, Canada, June 2004.

[19] P. Gorder, "Vortex drive," New Scientist, pp. 30-35, October 23-29 2004.

[20] K. Mohseni, "Zero-mass pulsatile jets for unmanned underwater vehicle maneuvering," in AIAA 3rd Unmanned Unlimited Technical Conference, AIAA Paper 2004-6386, Chicago, IL, September 2004.

[21] A. Polsenberg-Thomas, Michele, M. Grazier-G'Sell, K. Fisher, and J. Burdick, "Synthetic jet propulsion for small underwater vehicles," in Proc. IEEE Int. Conf. on Robotics and Automation, Barcelona, Spain, April 2005.

[22] C. Willert and M. Gharib, "Digital particle image velocimetry," Experiments in Fluids, vol. 10, pp. 181-193, 1991. 